\title{
Premarket Review
}

National Cancer Institute

\section{Source}

National Cancer Institute. Premarket Review. NCI Thesaurus. Code C54698.

The examination of data and information in an application for premarket review described in sections $505,510(\mathrm{k}), 513(\mathrm{f}), 515$, or $520(\mathrm{~g})$ or $520(\mathrm{l})$ of the Federal Food, Drug, and Cosmetic Act or section 351 of the Public Health Service Act of data and information contained in any investig ational new drug application, investig ational device exemption, new drug application, biologics license application, device premarket notification, device reclassification petition, and premarket approval application. 